BBA 43088

\title{
The stability of the two-bonded collagen triple helix
}

In the triple helical structure of collagen proposed by RAMACHANDRAN and co-workers $^{\mathbf{1}, 2}$ (see ref. 2 for earlier references), there are two NH...O hydrogen bonds for every three residues in each chain. In the modification of the RAMACHANDRANKARTHA structure proposed by RICH AND $\mathrm{CRICK}^{3}$, the three chains are pushed slightly farther apart from their positions in this two-bonded structure and only one set of hydrogen bonds is formed. The reason adduced for this further separation of the chains was that some of the atoms in neighbouring chains are impossibly close to one another in the two-bonded structure.

On the basis of the limiting contact distances ${ }^{4}$ which have been found to be in good agreement with a large number of observed polypeptide conformations $\mathbf{s}^{5,6}$, the two-bonded structure has been found to be perfectly permissible ${ }^{2}$. However, this is a purely qualitative statement. We have therefore calculated the Van der Waals interaction energy of the two structures. This calculation shows that the two-bonded structure has in fact a lower energy than the one-bonded structure, even with regard to the Van der Waals contribution to the energy, quite apart from the fact that it has one additional NH... O hydrogen bond for every three residues.

The Van der Waals potential functions used for the calculation were those proposed by Scheraga and co-workers ${ }^{7}$ and extended by BRANT AND Flory ${ }^{8}$. The numerical constants of the potential, of the form $V(r)=a \exp (-b r)-c / r^{6}$ were evaluated following the method described in ref. 8 . The coordinates of the backbone atoms and the two atoms of the side group attached to the $\alpha$ carbon atom (the two hydrogens for glycine and one hydrogen and the $\beta$ carbon atom for the other residues) were taken from ref. 2 for the two-bonded structure (called Structure A in this paper). For the one-bonded structure (Structure B), the coordinates of such a structure worked out at Madras ${ }^{9}$, corresponding to a value of $2.9 \mathrm{I} \AA$ for the unit height $h$ (the same as for the two-bonded structure) were employed. This structure is essentially similar to the one due to RICH AND CRICK ${ }^{3}$ (Structure B', which however has $h$ $=2.86 \AA$ ) and the earlier one-bonded structure published from Madras ${ }^{\mathbf{1 0}}$, with $h$ $=2.95 \AA$. The Van der Waals interaction energy was calculated including all neighbours of an atom which occur in residues separated by up to $\pm 9 \AA$ along the axis of the triple helix.

\section{TABLE I}

STABILISING ENERGY OF THE ONE-BONDED AND TWO-BONDED COLLAGEN HELICES The energy is expressed in $\mathrm{kcal} / \mathrm{mole}$ per residue.

\begin{tabular}{|c|c|c|c|}
\hline & \multirow{2}{*}{$\begin{array}{l}\text { Two-bonded structure } \\
h=2.9 I A \\
(\text { ref. } 2)\end{array}$} & \multicolumn{2}{|c|}{ One-bonded structure } \\
\hline & & $\begin{array}{l}h=2.9 I A \\
(\text { ref. } 9)\end{array}$ & $\begin{array}{l}h=2.86 A \\
(\text { ref. } 3)\end{array}$ \\
\hline Van der Waals energy & $-7 \cdot 5$ & -6.5 & -6.3 \\
\hline Hydrogen bond energy (approx.) & -3.7 & -1.7 & $-\mathbf{I} \cdot 7$ \\
\hline Sum & -11.2 & -8.2 & -8.0 \\
\hline
\end{tabular}


The results are shown in the first row of Table I. It will be seen from this that the total stabilising energy of the two-bonded structure per residue is about I kcal per residue mole lower than that of the one-bonded structure. This is not taking into account the additional stabilising energy from the extra hydrogen bond that is formed in the two-bonded structure. Assuming that the energy of an NH... O hydrogen bond is about $5 \mathrm{kcal} / \mathrm{mole}$ (refs. II, I2) and that of a $\mathrm{CH}$... O hydrogen bond is about $2 \mathrm{kcal} / \mathrm{mole}$ (ref. II) and that $60 \%$ of the amino hydrogens are bonded in the Structure $\mathrm{A}$ and $33 \%$ in Structure $\mathrm{B}$, the total difference in energy between the two Structures $\mathrm{A}$ and $\mathrm{B}$ is about $3 \mathrm{kcal} / \mathrm{mole}$.

These considerations also hold vis $a$ vis the two-bonded structure with $h$ $=2.9 \mathrm{I} \AA$ and the RICH AND CRICK one-bonded Structure $\mathrm{B}^{\prime}$ with $h=2.86 \AA$ the binding energy of the latter is even slightly higher than the one-bonded Structure $B$. Thus, it is obvious that, far from being impossibly close-packed, as has been criticised by RICH AND CRICK, the two-bonded structure brings neighbouring residues into a configuration of lower potential energy than the one-bonded structure. It is therefore clear that the collagen triple helix will take up the configuration $\mathrm{A}$ in all the regions of its structure, except those where, because of the occurrence of sequences like Gly-Pro-Hyp, two hydrogen bonds cannot be made.

It is very significant that the Van der Waals stabilising energy per residue of $-7.5 \mathrm{kcal} / \mathrm{mole}$ for the collagen triple helix is of the same order as that for conformations close to the $\alpha$ helix $\left(n=3.6, h=\mathrm{I} .5^{\circ} \AA\right)$, for which the same potential functions give a value of close to $-6.5 \mathrm{kcal} / \mathrm{mole}$. A study of the relative stabilities of the $\alpha$ helix, the $\beta$ structure and the triple helix is under way and will be reported separately.

Financial support to one of the authors (G.N.R.) from the U.S. National Science Foundation is gratefully acknowledged. This work was supported by U.S. Public Health Service Grant AM $02830-08$.

Biophysics Research Division Institute of

G. N. RAMACHANDRAN

Science and Technology, University of Michigan,

C. M. VENKatachalam Ann Arbor, Mich. (U.S.A.)

I G. N. Ramachandran and G. Kartha, Proc. Indian Acad. Sci. Sect. A, 45 (I955) I 24.

2 G. N. Ramachandran and V. Sasisekharan, Biochim. Biophys. Acta, Io9 (I965) $3 \mathbf{r}_{4}$.

3 A. Rich and F. H. C. CRick, J. Mol. Biol., 3 (I96I) 483.

4 G. N. Ramachandran, C. Ramakrishnan and V. Sasisekharan, J. Mol. Biol., 7 (I963) 95.

5 C. Ramakrishnan and G. N. Ramachandran, Biophys. $J ., 5$ (I965) 909.

6 S. J. Leach, G. Némethy and H. A. Scheraga, Biopolymers, in the press.

7 R. A. Scott and H. A. Scheraga, J. Chem. Phys., 42 (I965) 2209.

8 D. A. Brant and P. J. Flory, J. Am. Chem. Soc., 87 (I965) 2791.

9 G. N. Ramachandran, V. Sasisekharan, A. V. Lakshminarayanan, C. M. Ven Katachalam AND C. RAMAKRISHNAN, to be published.

io G. N. Ramachandran, V. Sasisekharan and Y. T. Thathachari, in N. Ramanathan, In Collagen, Interscience, New York, r96o, p. 8I.

i G. "C. Pimentel and A. L. McClellan, The Hydrogen Bond, W. H. Freeman, San Francisco, I 960, p. 362 .

i2 C. H. Bamford, A. Elliort and W. E. Hanby, Synthetic Polypeptides, Academic Press, New York, I956, p. I I I.

Received March IIth, I966 